\title{
Assessing Vignetting as a Means to Reduce VR Sickness During Amplified Head Rotations
}

\author{
Nahal Norouzi \\ University of Central Florida \\ Orlando, FL \\ nahal.norouzi@knights.ucf.edu
}

\author{
Gerd Bruder \\ University of Central Florida \\ Orlando, FL \\ bruder@ucf.edu
}

\author{
Greg Welch \\ University of Central Florida \\ Orlando, FL \\ welch@ucf.edu
}

\begin{abstract}
Redirected and amplified head movements have the potential to provide more natural interaction with virtual environments (VEs) than using controller-based input, which causes large discrepancies between visual and vestibular self-motion cues and leads to increased VR sickness. However, such amplified head movements may also exacerbate VR sickness symptoms over no amplification. Several general methods have been introduced to reduce VR sickness for controller-based input inside a VE, including a popular vignetting method that gradually reduces the field of view.

In this paper, we investigate the use of vignetting to reduce VR sickness when using amplified head rotations instead of controllerbased input. We also investigate whether the induced VR sickness is a result of the user's head acceleration or velocity by introducing two different modes of vignetting, one triggered by acceleration and the other by velocity. Our dependent measures were pre and post VR sickness questionnaires as well as estimated discomfort levels that were assessed each minute of the experiment. Our results show interesting effects between a baseline condition without vignetting, as well as the two vignetting methods, generally indicating that the vignetting methods did not succeed in reducing VR sickness for most of the participants and, instead, lead to a significant increase. We discuss the results and potential explanations of our findings.
\end{abstract}

\section{CCS CONCEPTS}

- Human-centered computing $\rightarrow$ User studies; Virtual reality;

\section{KEYWORDS}

Simulator Sickness, VR Sickness, Amplified Head Rotations, Vignetting, Virtual Environments, Applied Perception

\section{ACM Reference Format:}

Nahal Norouzi, Gerd Bruder, and Greg Welch. 2018. Assessing Vignetting as a Means to Reduce VR Sickness During Amplified Head Rotations. In SAP '18: ACM Symposium on Applied Perception 2018, August 10-11, 2018, Vancouver, BC, Canada. ACM, New York, NY, USA, 8 pages. https://doi.org/ $10.1145 / 3225153.3225162$

Permission to make digital or hard copies of all or part of this work for personal or classroom use is granted without fee provided that copies are not made or distributed for profit or commercial advantage and that copies bear this notice and the full citation on the first page. Copyrights for components of this work owned by others than ACM must be honored. Abstracting with credit is permitted. To copy otherwise, or republish, to post on servers or to redistribute to lists, requires prior specific permission and/or a fee. Request permissions from permissions@acm.org.

SAP '18, August 10-11, 2018, Vancouver, BC, Canada

(C) 2018 Association for Computing Machinery.

ACM ISBN 978-1-4503-5894-1/18/08 ..\$15.00

https://doi.org/10.1145/3225153.3225162

\section{INTRODUCTION}

Perception and cognition research has shown benefits of self-motion inside a virtual reality (VR) environment using a natural, multimodal method like head-tracked movements compared to other interaction techniques, such as flying or steering with controllerbased input [Steinicke et al. 2013]. VR sickness causes symptoms similar to motion sickness in the real world, including headaches, nausea, vomiting, sweating, fatigue, drowsiness, and disorientation [Kennedy et al. 1993]. The most commonly accepted explanation for VR sickness is the sensory conflict theory, describing conflicts that arise when visual self-motion cues do not match the cues provided by the vestibular and proprioceptive system [Reason and Brand 1975]. For instance, when users navigate through a virtual environment (VE) using a hand-held controller while their head and body remain stationary in the real world, the visual cues indicate self-motion, whereas the vestibular and proprioceptive systems indicate that they are not moving, which introduces a sensory conflict that can cause VR sickness [McCauley and Sharkey 1992]. Moreover, controller-based input is known to have further drawbacks such as a reduction in the user's sense of presence [Robinett and Holloway 1992; Slater et al. 1995], which denotes the illusion of "being there" in the VE [Schuemie et al. 2001; Slater et al. 1994].

However, it is not always convenient or possible to rely on natural head movements alone, such as when a user's range of head movements in the physical environment is restricted due to physical support, the range of tracking sensors, or limited interaction space. Different methods have been introduced to address this challenge. Popular approaches are based on manipulating the user's virtual rotation and/or translation compared to their real movements with techniques including redirected walking [Razzaque et al. 2001; Steinicke et al. 2010] and amplified head rotations [Jay and Hubbold 2003; LaViola, Jr. et al. 2001; Ragan et al. 2017]. While empirical evidence for a correlation between amplified head rotations and VR sickness is scarce, the notion is largely accepted that such methods can also cause occurrences of VR sickness, although at a comparatively lower strength than controller-based input [Nilsson et al. 2018; Steinicke et al. 2013].

Mainly focusing on controller-based input, both in academia and the gaming industry, researchers have introduced methods for reducing VR sickness. In the gaming industry, the term comfort mode is often used to denote these methods, which include a gradual reduction in the field of view (FOV) [Bolas et al. 2017; Fernandes and Feiner 2016], sometimes called vignetting, as well as blurring of the view [Budhiraja et al. 2017] and rotation snapping techniques [Farmani and Teather 2018]. A recent study has shown that the vignetting method can greatly reduce the observed VR sickness when using controller-based input with a low impact on 
the user's sense of presence [Fernandes and Feiner 2016]. Moreover, a similar vignetting method based on a recent patent [Bolas et al. 2017] is leveraged when using controller-based input in Google Earth VR, although this application does not use vignetting during natural head rotations. It remains a challenging question whether these VR sickness reduction techniques are applicable for reducing sickness caused by (amplified) head movements or only when using controller-based input.

In this paper we investigate the effects of vignetting as a means to reduce VR sickness when using amplified head rotations instead of controller-based input. Considering that head rotations consist of different phases, with acceleration and velocity components, in contrast to controller-based button or joystick input, we describe two vignetting methods in this paper based on acceleration and velocity. We evaluated the vignetting methods and compared them against a baseline condition in terms of their effects on VR sickness, presence, user preference, and performance.

\section{RELATED WORK}

In this section, we provide an overview of related work on redirected or amplified head movements and VR sickness.

\subsection{Amplified Head Movements}

A large body of literature has focused on developing methods to change a user's self-motion feedback in a VE compared to natural head movements in the real world [Nilsson et al. 2018]. One of the first approaches in this field was proposed by Razzaque et al., who introduced redirected walking [Razzaque et al. 2001] as a means to guide users on paths in the real world that differed from the visually perceived paths by introducing slightly nonisomorphic rotations of the virtual camera in response to physical head movements [LaViola, Jr. et al. 2001]. While many of these methods assume that the user is physically walking, some of these methods can also be applied to stationary users, e.g., while standing or sitting [Ragan et al. 2017; Razzaque et al. 2002; Sargunam et al. 2017]. For instance, Razzaque et al. introduced a method to prevent users from facing an open back wall when standing in a CAVE-like setup by rotating the VE undetectably opposite to the user's view direction so that the user would always eventually turn toward the front wall. In a similar attempt, LaViola et al. explored amplifying a user's head and torso rotations in the VE compared to the real world using a linear scaling factor, such that a 120-degree physical rotation was mapped to a virtual 180-degree rotation, and they later extended such amplified head rotations with nonlinear mappings [LaViola, Jr. et al. 2001]. Sargunam et al. proposed an improved method called guided head rotations for seated users, which included a realignment technique on top of the amplification, aimed at bringing the user back to a comfortable head/neck orientation [Sargunam et al. 2017].

In the context of these methods, important topics such as the detectability of the rotation amplification and its relationship with different FOVs has been the focus of several researchers [Bolte et al. 2010; Kopper et al. 2011; Ragan et al. 2017; Zhang and Kuhl 2013]. Bolte et al. investigated the effects of amplified head rotations and FOV augmentation on comfort and detectability when performing pitch and roll rotations, which showed that even large differences went unnoticed by users, and results from presence and VR sickness questionnaires seemed to be unaffected by the method [Bolte et al. 2010]. Ragan et al. investigated the effects of amplified head rotations on spatial orientation, VR sickness, and 3D search tasks among different amplification gains and display options [Ragan et al. 2017]. Their experiment demonstrated the feasibility of using amplified head rotation in VR, but noticeable issues were identified when using strong amplification gains. In particular, they found a significantly higher VR sickness for amplified head rotations when using a head-mounted display (HMD) than for a CAVE-like setup.

\subsection{VR Sickness and Reduction Techniques}

VR sickness, or cybersickness, has many similarities to simulator sickness and motion sickness and although different people experience it differently based on their level of susceptibility, it mostly presents itself through symptoms such as eye strain, stomach awareness, dizziness, nausea, and headaches among others [Kennedy et al. 1993; McCauley and Sharkey 1992; Rebenitsch and Owen 2014; Stanney et al. 1997], some of which are related to VR display technologies [Kellnhofer et al. 2016; Koulieris et al. 2016]. Factors such as age, gender and illness might separately affect one's level of susceptibility to VR sickness [Biocca 1992; Frank et al. 1984; Reason and Brand 1975]. In a study by Kennedy et al., effects of duration and repetition of exposure on simulator sickness were measured by examining 938 and 53 cases, respectively [Kennedy et al. 2000]. Results showed that simulator sickness is related to duration and repetition of exposure, the former having a negative effect and the latter a positive effect. Based on similar observations [Kennedy et al. 2000; McCauley and Sharkey 1992], different methods inspired by exposure therapy, e.g., using repeated exposures with optokinetic training [Ressiot et al. 2013], have been proposed to reduce motion sickness in the real world.

Although repeated exposure may reduce the effects of VR sickness, there is a large demand from the gaming industry and professional VR applications to make first-time experiences with VR technologies more comfortable and less sickening. One factor that has been found to be directly related to the level of VR sickness experienced by users is the FOV and display technology used [Lin et al. 2002; Patrick et al. 2000; Prothero 1995; Ragan et al. 2017; Sharples et al. 2008; Stanney and Kennedy 1997]. In particular, the FOV of HMDs has been extensively researched, which has revealed that a smaller visual field is positively correlated with a reduction in VR sickness, while it is also correlated with a reduction in the user's sense of presence. Hence, instead of just overall reducing the FOV, Bolas et al. patented the approach to gradually reduce the FOV only in situations that are known to cause VR sickness, such as when using controller-based input to navigate through a VE [Bolas et al. 2017].

Fernandes and Feiner evaluated the approach, which is also known as vignetting, with a human-subject study. Participants explored a VE using a hand-held controller while the vignetting-based reduction in FOV was controlled by factors including the angular velocity and overall speed up to a predefined maximum amount. They measured the participants' VR sickness and discomfort using a so-called discomfort score and related question [Rebenitsch and Owen 2014]. They found a significant reduction in VR sickness and discomfort when using the vignetting method with controllerbased input. Introducing a similar method as vignetting, Budhiraja 
et al. proposed rotation blurring, which increased or decreased the amount of Gaussian blur applied to user's view, depending on the accelerations of the movements of a computer mouse [Budhiraja et al. 2017]. In their study, 8 out of 15 participants significantly benefited from the rotation blurring method.

Of the two methods described above, the former was controlled by the velocity and the latter by the acceleration of virtual camera rotations. So far, it is not clear how much of a role velocity and acceleration play in the induced level of VR sickness during rotations in VR. Some research looked into effects of velocity and acceleration on VR sickness with anecdotal results that suggested that one or the other might have exacerbated the levels of the sickness. For instance, in a study performed with driving simulators, they found that sickness levels increased in rural and highway environments where users could drive $60 \mathrm{mph}$ unlike city environment where the speed was limited to $25 \mathrm{mph}$ [Mourant and Thattacherry 2000]. They concluded based on their results that velocity might have an effect on sickness levels. In a different study, Bonato et al. exposed participants to either a steadily expanding full-field optic flow pattern or a constantly expanding and contracting pattern. The results of that study showed that in the steady expansion group the levels of sickness experienced were significantly lower [Bonato et al. 2008], which might suggest a potential effect of acceleration on sickness levels. In a different study focused on reducing VR sickness with a method of proprioceptive vibrations, Plouzeau et al. suggested that VR sickness may be exacerbated by both high velocity and acceleration during movements [Plouzeau et al. 2015].

Based on these anecdotal results, we were interested in investigating if velocity or acceleration-based vignetting during amplified head rotations would have a significant effect on VR sickness.

\section{IMPLEMENTATION}

In this section, we describe the method of amplified head rotations as well as our adapted methods of velocity and acceleration-based vignetting, which are used in our experiment.

\subsection{Amplified Head Rotations}

A rotation gain $g_{\psi} \in \mathbb{R}$ for yaw rotations $\psi$ is defined by the quotient of the considered component of a virtual yaw rotation $\psi_{\text {virtual }}$ and the real-world yaw rotation $\psi_{\text {real }}$, i.e., $g_{\psi}=\frac{\psi_{\text {virtual }}}{\psi_{\text {real }}}$. When a rotation gain $g_{\psi}$ is applied to a real-world yaw rotation $\psi_{\text {real }}$, the virtual camera is rotated by $\psi_{\text {real }} \cdot g_{\psi}$ instead of $\psi_{\text {real }}$. This means that if $g_{\psi}=1$, the virtual scene remains stable considering the head's orientation change. In the case $g_{\psi}>1$, the virtual camera rotates faster than the user's head, whereas a gain $g_{\psi}<1$ causes the virtual camera to rotate slower. For instance, if a gain $g_{\psi}=2$ is applied and the user rotates the head 90 degrees in the real world, the virtual camera is rotated by 180 degrees [Steinicke et al. 2010]. In our experiment, we used amplified rotations with such gains only for yaw head rotations, not for pitch or roll rotations [Bolte et al. 2010].

\subsection{Velocity-Based Vignetting}

To implement the velocity-based vignetting method, we followed the approach proposed by Fernandes and Feiner [Fernandes and Feiner 2016], and adapted it to scale depending on head rotation velocity, not controller-based input. We first defined the minimum and maximum amount of FOV restriction. The minimum restriction of the FOV was set to 0 degrees and the contraction of the vignette should reach the maximum as in Fernandes and Feiner [Fernandes and Feiner 2016], resulting in a FOV of 80 degrees. Inspired by and similar to [Budhiraja et al. 2017], we took a modal approach for our implementation, meaning the minimum amount was assumed when the user's angular velocity fell below a velocity threshold $\tau_{v} \in \mathbb{R}^{+}$, so that the according vignetting would not be triggered for very small movements that might distract the user. Fernandes and Feiner [Fernandes and Feiner 2016] and Sargunam et al. [Sargunam et al. 2017] also incorporated the capability for such thresholds in their computational models, which result in relatively little impact on the user's view and amplification rate while small head motions are made and cap the maximum vignetting such that the view to the VE is never blocked entirely. After performing several pilot tests with 10 participants taking into account both larger and smaller head rotations and analyzing their head velocity profiles, we chose a threshold of $\tau_{v}=20 \mathrm{deg} / \mathrm{s}$. Figure 1 shows a sample head rotation which takes less than one second from one stationary state to the next. The higher the angular velocity, the faster the vignette would reach its maximum. We used the formula $r_{c}=v_{a} \cdot s$ to compute the rate of contraction $r_{c} \in \mathbb{R}^{+}$based on the angular velocity $v_{a}$ in $\mathrm{deg} / \mathrm{s}$ being computed every frame and a scaling factor $s \in \mathbb{R}^{+}$(set to $s=0.2$ after pilot tests).

\subsection{Acceleration-Based Vignetting}

For the implementation of the acceleration-based vignetting method, we focused on the onset and offset of the user's head rotation, i.e., the moment when they start their head rotation and the moment before they stop, which for head rotations usually happen in less than a second. We defined an onset and offset acceleration threshold of $\tau_{a} \in \mathbb{R}^{+}$while keeping track of the increase or decrease in acceleration and classified the user's head rotation state as either stationary or non-stationary to distinguish between the onset and offset of head accelerations. As for velocity-based vignetting, we determined a reasonable threshold using a pilot study, which resulted in our choice of $\tau_{a}=15 \mathrm{deg} / \mathrm{s}^{2}$. The contracting vignettes would appear for roughly $100 \mathrm{~ms}$ to $150 \mathrm{~ms}$ on the onset and offset of the user's head rotations, thus visually masking the two acceleration and deceleration phases after and before going in the stationary states. Figure 1(c) shows a sample head rotation with acceleration-based masking, corresponding to the sample head motion and thresholds shown in Figure 1(a).

\section{EXPERIMENT}

In this section, we describe the experiment that we conducted to compare acceleration and velocity-based vignetting with a control condition in terms of their effects on VR sickness, presence, user preference, and performance.

\subsection{Participants}

Overall, 18 participants (twelve male, six female, age 19-24, average 21.72) were recruited and participated in all three sessions of the experiment. All participants were affiliated with our institution. 14 participants were undergraduate students, three were graduate students, and one recently graduated. Only two of the participants mentioned that they had used an HMD more than ten times before. None of the participants reported any known visual disorder such 


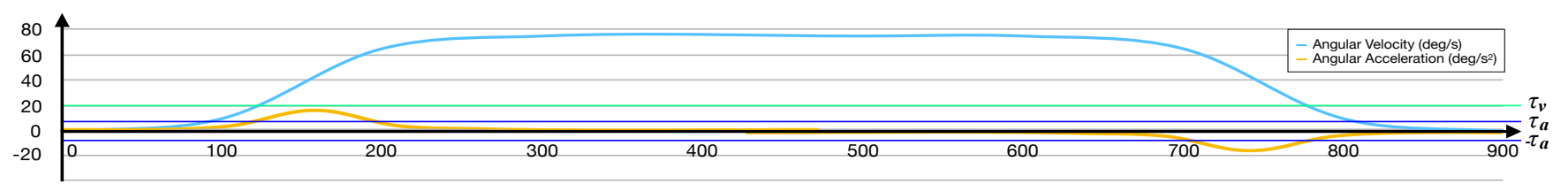

(a)

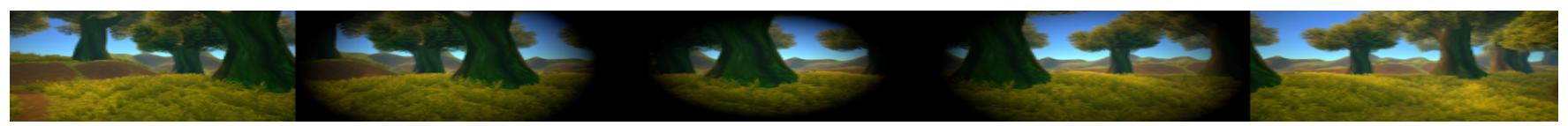

(b)

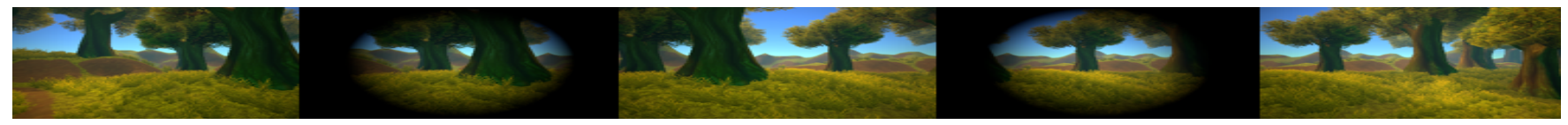

(c)

Figure 1: Illustration of the implementation of the vignetting methods for the (a) sample angular velocity pattern of a head rotation from one stationary state to the next. The thresholds indicate the points where the methods based on head rotation (b) velocity or (c) acceleration introduce the vignetting effects to the visual field.

as stereo blindness, night blindness, or color blindness. Moreover, none of them reported a disorder of equilibrium. Participants were asked to judge their susceptibility to motion sickness on a scale of 1 (not at all) to 5 (very susceptible), which revealed an average susceptibility of $1.5(S D=0.7, \min =1, \max =3)$.

Therefore, we asked them to rate their status before donning the HMD on a scale of 0 to 10 , with 0 meaning that they were feeling perfectly fine and 10 meaning that they were feeling stressed, exhausted, and overloaded. Two of our participants scored 8 on this rating scale, which we excluded from our analysis. The reasoning behind this exclusion was that they reported the reason they felt disorientated during one of the conditions was due to their own exhaustion before coming in and not because of the method they experienced, while they actually preferred that method overall. Also, one participant was extremely sensitive to the VE and suffered from strong postural instability in all conditions. Hence, we had to remove these participants, and the results reported are for the remaining 15 participants.

\subsection{Material}

The experimental setup consisted of an HTC VIVE HMD, featuring a resolution of $2160 \times 1200$ pixels $(1080 \times 1200$ per eye), a refresh rate of $90 \mathrm{~Hz}$, a nominal field of view of 110 degrees, and a weight of 470 grams. The HTC Lighthouse tracking system was used for the position and orientation tracking of the HMD. A graphics workstation with an Intel Xeon $2.4 \mathrm{GHz}$ processor comprising 16 cores, $32 \mathrm{~GB}$ of main memory and two Nvidia Geforce GTX 980 Ti graphics cards was used for system control, rendering, and logging. Figure 2 illustrates the experimental setup.

The application was developed in the Unity 2017.3.1 graphics engine. For the VE, we incorporated the Hand Painted Forest asset from the Unity Asset Store. As a means to communicate rotation tasks to the participants we used a virtual butterfly as a visual target, similar to the butterfly used by Peck et al. in related research [Peck et al. 2008]. The VE with the virtual butterfly is shown in Figure 3.

\subsection{Methods}

In this experiment, we used a within subject design with the method of constant stimuli. The experiment was conducted over two sessions which lasted for three consecutive days each.

We compared the following three conditions:

C Control condition without vignetting.

V Velocity-based vignetting technique.

A Acceleration-based vignetting technique.

We randomized the order of the conditions over three consecutive days to compensate for any potential effects related to a reduction in VR sickness caused by repeated exposure [Kennedy et al. 2000; McCauley and Sharkey 1992].

4.3.1 Measures. We used the following measures in this experiment to identify differences in VR sickness, discomfort, presence, preference, and task performance.

SSQ To measure the participants' levels of VR sickness we used the Simulator Sickness Questionnaire (SSQ) [Kennedy et al. 1993], which they filled out before and after each condition.

DS In line with Fernandes and Feiner [Fernandes and Feiner 2016], we used a similar version of the Discomfort Score (DS) to evaluate the participants' sense of discomfort after each minute during the experience. Therefore, a message appeared asking them "On a scale of 0 to 10, 0 being how you felt coming in, 10 is feeling nauseous and wanting to stop, where are you now?" [Fernandes and Feiner 2016; Rebenitsch and Owen 2014; Teasdale et al. 2009].

SUS To measure the participants' sense of presence, they filled out the Slater-Usoh-Steed (SUS) presence questionnaire [Usoh et al. 2000] after each condition.

PR After the final session, we asked them to indicate their preferred method of interaction and rank the three methods.

PE We tracked the percentage of time when each participant was able to see the butterfly in their visual field, which is an 


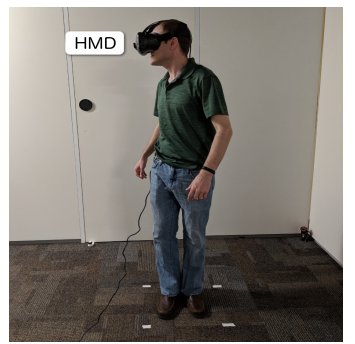

Figure 2: Photo of the physical setup with a marked position for the participants to stand in with a fixed orientation of their feet, while wearing an HTC VIVE HMD.

objective measure of whether the conditions had an effect on the participants' task performance.

We further collected qualitative feedback after the experiment and asked the participants to give their impression of what might have caused their feelings of discomfort during the experiment.

4.3.2 Procedure. At the beginning of the experiment, participants were guided into the experimental space. There, they read a summary of the task they were asked to perform, the task was explained to them, and they saw a short preview of the task before they started. After they gave their informed consent, they filled out a demographics questionnaire and the pre-SSQ.

Participants were instructed to stand inside a predefined $50 \mathrm{~cm}$ by $50 \mathrm{~cm}$ area (see Fig. 2), and put on the tracked HMD. They were informed that they could rotate their head and their torso but they should not move their feet, which we introduced to avoid unintentional positional drift during the experiment. By asking participants to stand and not sit during the experiment, we made sure that postural stability effects brought about by a seated user were not having an (in this study) unintended positive effect on their level of VR sickness [Riccio and Stoffregen 1991].

Once they donned the HMD, they saw a VE consisting of a virtual forest scene with a virtual butterfly in front of them. Participants were instructed to follow the butterfly which was moving around them in a circular horizontal motion at a distance of 1.5 meters. The movements of the butterfly were pre-defined so that every participant experienced the same moving target sequence. However, the butterfly's moving pattern was highly dynamic and designed in a way so that it would be very hard to memorize, and with the goal that the participants would have to make frequent and large (physical and virtual) head rotations in order to follow the butterfly.

A gain of $g_{\psi}=3$ was applied to head yaw rotations in all three conditions, which means that a physical rotation of 60 degrees in the real world was mapped to a virtual camera rotation of 180 degrees in that direction [Steinicke et al. 2010], thus allowing the participants to view the entire 360-degree virtual space with head rotations alone, i.e., without having to rotate their physical body. While previous work suggests that variable gains could be used to be less noticeable to users, in this study we decided to control this factor and apply a comparably strong constant gain to elicit noticeable sensory conflicts between the visual and vestibular system for all users independently of their individual sensitivity threshold.

After each minute in the experiment, we asked them to answer the discomfort score question by verbally indicating a number

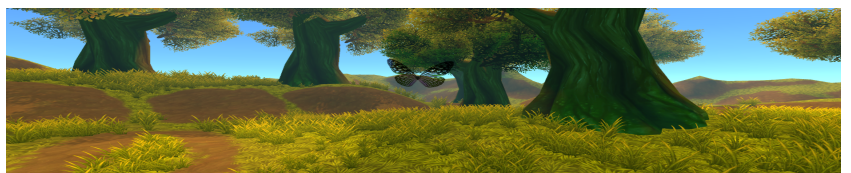

Figure 3: Screenshot of the virtual environment used in the experiment. The participants' task was to follow the butterfly that was moving around them.

between 0 and 10. Participants were asked to stay in the VE and follow the butterfly as best as they can.

However, once their discomfort reached the maximum score of 10 , they were asked to stop and take off the HMD. Alternatively, after 20 minutes without reaching the maximum discomfort score, the session ended automatically. After the session, they were asked to fill out the post-SSQ and SUS questionnaires. After the last session, they were further asked to indicate their preferred method and provide qualitative feedback about the methods and their feelings of discomfort.

4.3.3 Hypotheses. In this experiment, we evaluated the following hypotheses:

$\mathrm{H}_{1}$ In both conditions $\boldsymbol{V}$ and $\boldsymbol{A}$ we will see lower sickness scores compared to the baseline condition $\boldsymbol{C}$. Vignetting has been shown to reduce VR sickness when using controller-based input [Fernandes and Feiner 2016], such that we expected to see benefits of using both velocity and acceleration-based vignetting techniques for amplified head rotations as well.

$\mathrm{H}_{2}$ Sickness scores will be lower in condition $\boldsymbol{A}$ than $\boldsymbol{V}$. It is reasonable to assume that the onset and offset phases of head rotations may have a stronger effect on VR sickness, e.g., due to latency or oculomotor reflexes, such that the accelerationbased method could mask them more effectively.

$\mathrm{H}_{3}$ Presence scores will be highest in condition $C$, followed by condition $\boldsymbol{A}$, and finally condition $\boldsymbol{V}$. Considering that vignetting may intrude in the participant's sense of presence, we expected to see a stronger degraded presence the more vignetting was applied.

$\mathrm{H}_{4}$ Participants will prefer condition $\boldsymbol{C}$. It seems reasonable to assume that they would prefer the baseline condition as the most natural way of interaction among the three conditions.

$\mathrm{H}_{5}$ Task performance will be highest in condition $\boldsymbol{C}$. We expected task performance to be higher when no vignettes are applied.

\section{RESULTS}

Considering the large individual differences that were to be expected in susceptibility to simulator sickness, we also expected differences in how the sickness reduction techniques would be received by the participants. Recognizing that VR sickness is a highly individual phenomenon and that no technique might work for all participants, Budhiraja et al. [Budhiraja et al. 2017] proposed a method to focus on those groups that would gain benefits or detriments due to the tested techniques. In this paper, we follow their approach. In the following, we describe the analysis of the discomfort scores of the 15 participants, and we found that the majority of them (11 participants) had the lowest discomfort scores in the baseline condition $\mathrm{C}$, whereas the others (4 participants) had 


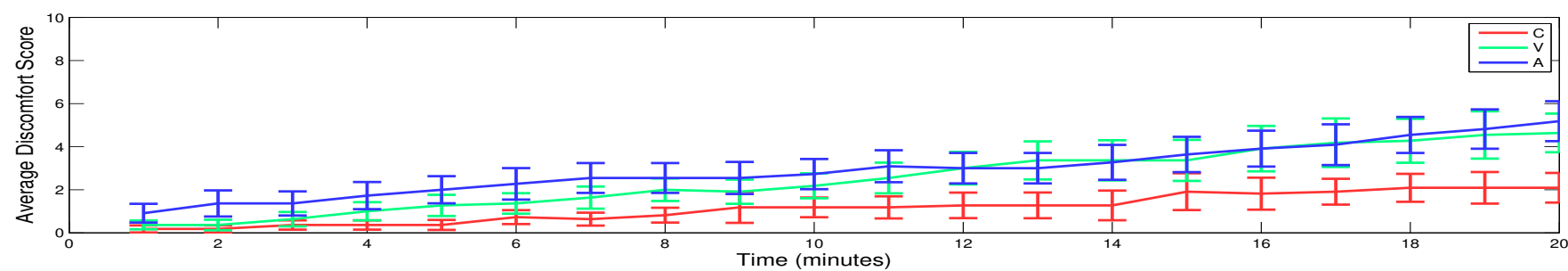

Figure 4: Discomfort Scores pooled over all participants for the three conditions C, V, and A during a 20 minute session.

lower scores in either of the vignetting conditions $\mathrm{V}$ or $\mathrm{A}$. We thus decided to split the analysis into these two groups:

- Group 1 with 11 participants showed no benefits but detriments in terms of discomfort when using vignetting.

- Group 2 with 4 participants showed benefits related to discomfort when using vignetting in condition $\mathrm{V}$ and/or $\mathrm{A}$.

We analyzed the questionnaire data with non-parametric Friedman tests and Wilcoxon signed-rank post-hoc tests with Bonferroni correction for multiple comparisons.

\subsection{Discomfort Scores}

Figure 4 shows the pooled Discomfort Scores over all participants for each minute in the three conditions. For the analysis of the Discomfort Scores (DS), we used a derived measure called Relative Discomfort Scores (RDS), which was introduced by Fernandes and Feiner [Fernandes and Feiner 2016]. Using this measure, each minute in the experiment after a participant drops out due to a discomfort score of 10 will be counted as an additional 10. As discussed by Fernandes and Feiner, this analysis has the advantage that it takes into account the duration that participants actually spent in the environment and gives penalties for dropping out early. Out of the 15 participant data sets in our analysis, ten of them experienced all three conditions for the full duration of 20 minutes, and those five who terminated early stayed in the environment for at least 14 minutes. Figure 5(a) shows the pooled RDS results for the groups. Figure 6(a) shows the RDS for each participant for each condition; the participants to the left of the red line belong to Group 1, the others to Group 2.

We found a significant main effect of condition on RDS for Group 1; $\chi_{r}^{2}(2)=14.36, p<0.001$; but not for Group 2; $\chi_{r}^{2}(2)=$ $0.5, p=0.78$. We conducted pairwise comparisons for Group 1 . We found significant differences in RDS between conditions $\mathrm{C}$ and $\mathrm{V}$ $(w=0, Z=-2.666, p=0.01, r=0.880)$ as well as between conditions $\mathrm{C}$ and $\mathrm{A}(w=0, Z=-2.934, p=0.02, r=0.885)$. We did not find significant differences when comparing $\mathrm{V}$ with $\mathrm{A}(w=27.0$, $Z=-0.533, p=0.59, r=0.161)$.

\subsection{Simulator Sickness Questionnaire Scores}

We analyzed the SSQ responses by computing the pre and post SSQ scores (measured before and after each condition) and subtracting them from each other to compute the relative increase (or decrease) during the condition. Additionally to computing the Total (T) scores, we computed the results for the three SSQ sub-scales: Nausea (N), Oculomotor (O), and Disorientation (D). Figure 5(b) shows the pooled SSQ results for the groups. Figure 6(b) shows the Total
Table 1: Main effects and post-hoc tests for the SSQ Total (T) scores and sub-scales Nausea (N), Oculomotor (O), and Disorientation (D) for Group 1.

\begin{tabular}{|c|c|}
\hline $\mathrm{T}$ & $\chi_{r}^{2}(2)=\mathbf{1 2 . 6 8 2}, \boldsymbol{p}=\mathbf{0 . 0 0 2}$ \\
$\mathrm{N}$ & $\chi_{r}^{2}(2)=\mathbf{9 . 1 3 6 , p}=\mathbf{0 . 0 1}$ \\
$\mathrm{O}$ & $\chi_{r}^{2}(2)=\mathbf{9 . 5 9 1}, \boldsymbol{p}=\mathbf{0 . 0 1}$ \\
$\mathrm{D}$ & $\chi_{r}^{2}(2)=5.636, p=0.06$ \\
\hline
\end{tabular}

\begin{tabular}{|c|c|c|c|c|}
\hline & $\mathrm{T}$ & $\mathrm{N}$ & $\mathrm{O}$ & $\mathrm{D}$ \\
\hline $\mathrm{C}$ vs. $\mathrm{V}$ & $p=0.20$ & $p=0.59$ & $\boldsymbol{p}=\mathbf{0 . 0 2}$ & $p=0.29$ \\
$\mathrm{C}$ vs. $\mathrm{A}$ & $\boldsymbol{p}=\mathbf{0 . 0 1}$ & $\boldsymbol{p}=\mathbf{0 . 0 2}$ & $p=0.25$ & $\boldsymbol{p}=\mathbf{0 . 0 4}$ \\
V vs. A & $p=0.08$ & $p=0.13$ & $p=0.25$ & $p=0.21$ \\
\hline
\end{tabular}

scores for all 15 participants; the participants to the left of the red line belong to Group 1, the others to Group 2.

As shown in Table 1, we found significant main effects and posthoc tests for the SSQ scores for Group 1. In particular, we found a significant main effect for the Total scores, the $\mathrm{N}$ and $\mathrm{O}$ sub-scales, as well as a trend for the D sub-scale. We found no significant effects for Group 2 for the Total scores; $\chi_{r}^{2}(2)=2.533, p=0.28$; or any of the sub-scales.

\subsection{Presence}

The results showed different SUS presence scores for condition C $(M=31.60, S D=5.527)$, condition V $(M=28.67, S D=5.473)$, and condition $\mathrm{A}(M=27.20, S D=8.091)$. We found a significant main effect of condition on SUS presence scores for Group $1 ; \chi_{r}^{2}(2)=$ 6.046, $p=0.049$; but not for Group 2; $\chi_{r}^{2}(2)=4.133, p=0.13$.

Post-hoc tests for Group 1 did not reveal significant differences for the presence scores between conditions $\mathrm{C}$ and $\mathrm{V}(w=40, Z=$ -1.274, $p=0.25, r=0.402)$ and between conditions $\mathrm{V}$ and $\mathrm{A}$ $(w=35.5, Z=-1.540, p=0.25, r=0.513)$, but a trend between conditions $\mathrm{C}$ and $\mathrm{A}(w=41.5, Z=-2.251, p=0.07, r=0.750)$.

\subsection{Preference}

All 15 participants were asked to choose their preferred method. Condition $\mathrm{C}$ was the preferred method of 12 participants and condition $\mathrm{V}$ was the preferred method of the remaining 3.

\subsection{Task Performance}

We computed the percentage of time the butterfly was in the participants' visual field in condition C $(M=68.91 \%, S D=10.59 \%)$, condition $\mathrm{V}(M=70.94 \%, S D=11.03 \%)$, and condition $\mathrm{A}(M=71.25 \%$, $S D=12.71 \%$ ), which provides an objective measure of how much the different conditions affected the participants' task performance. We found no significant main effect of the conditions on this measure of task performance; $\chi_{r}^{2}(2)=1.233, p=0.54$. 




Group \#

(a)
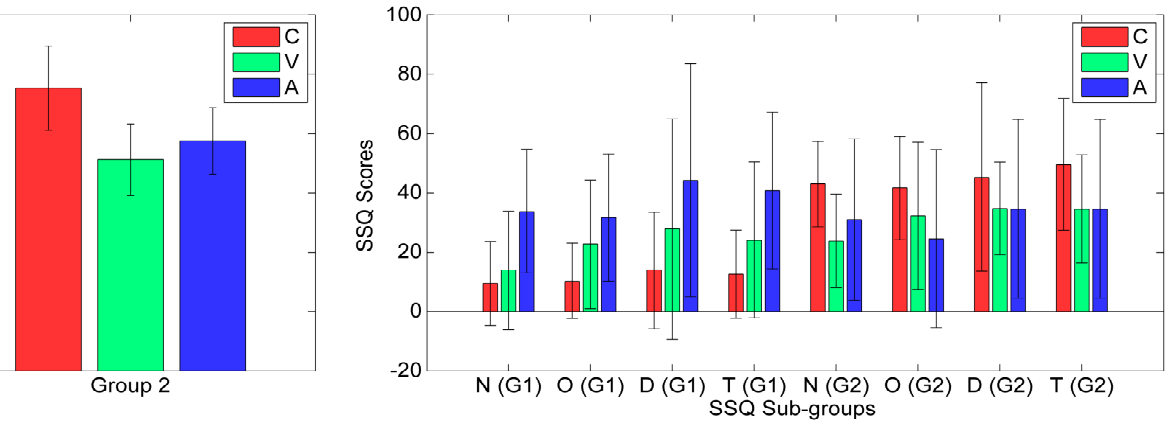

(b)

Figure 5: Pooled results for (a) the RDS for the two groups, and (b) the SSQ sub-scales Nausea (N), Oculomotor (O), and Disorientation (D) as well as the Total (T) scores among participants of Group 1 (G1) and Group 2 (G2).

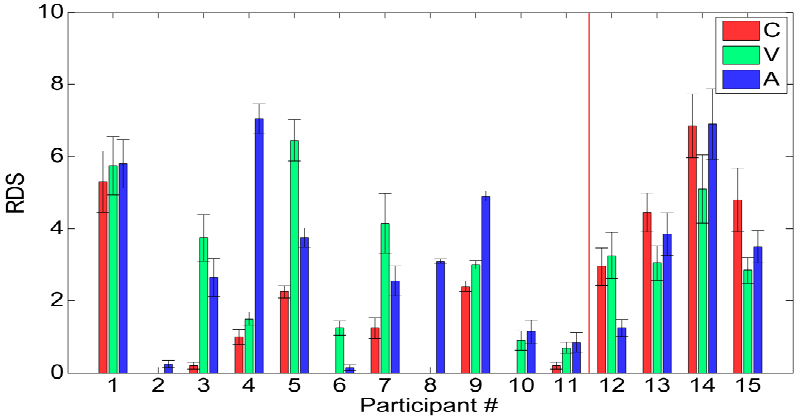

(a)

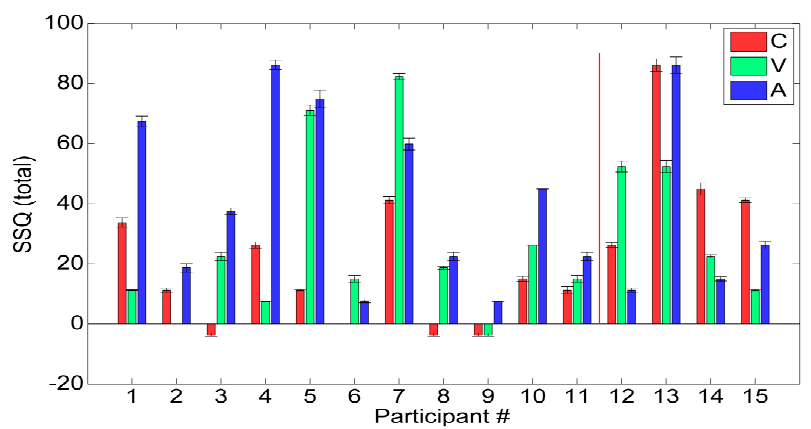

(b)

Figure 6: Individual results for all participants for (a) RDS and (b) SSQ scores for the three conditions. Participants 1-11 in Group 1 are separated from participants 12-15 in Group 2 by the red line.

\section{DISCUSSION}

We were surprised to see that the majority of participants (11 of 15 in total) experienced significantly more VR sickness if the velocity or acceleration-based vignetting methods were used than in the control condition. This result suggests the opposite effect compared to our Hypothesis $\mathrm{H}_{1}$, and it stands in contrast to the related work on vignetting, which was performed with controller-based input instead of amplified head rotations. We believe this is likely related to differences in how we explore and scan a VE using our head compared to using a hand-held controller. Head movements are naturally faster than using controllers during rotations, potentially hundreds of degrees per second, and on average begin and end in less than a second. In the case of hand-held controllers, rotation is sometimes mapped to the controller's orientation, and one has to physically maneuver their hands (the controller) to scan their environment. This hand-controller manipulation is typically slower than head rotation. Other times rotation is mapped to buttons on the controller, such that rotations are affected by pressing and holding a button until the desired rate or orientation has been reached. This too is typically much slower than head rotations. In either case, the associated gradual contraction of the vignetting during the (slower) rotation loses its effect and/or can tend to reach its maximum intensity to block the optic flow in the periphery as fast as possible. Based on our results, we can not recommend using vignetting as the default method for all users in VR when using head movements instead of controllers as input.

We did not find support for Hypothesis $\mathrm{H}_{2}$. The discomfort and sickness scores showed no significant differences between the velocity and acceleration based vignetting conditions, even for the group of participants that actually benefited from vignetting.

We only found limited support for Hypothesis $\mathrm{H}_{3}$, with a significant main effect of the conditions on subjective self-reports of presence, but no significant pairwise comparisons.

As expected, the majority of our participants (12 of 15 in total) preferred the control condition, where no FOV changes were introduced during interaction, which supports our Hypothesis $\mathrm{H}_{4}$.

We did not find an effect of the conditions on task performance, which does not provide support for Hypothesis $\mathrm{H}_{5}$.

As an interesting anecdotal observation, during the debriefing sessions we learned that none of our participants noticed that their head rotations were amplified. While previous results in the literature on amplified head rotations suggested that a certain magnitude of rotation gains could go unnoticed [Steinicke et al. 2010], we were surprised that this would go as high as to a gain of $g_{\psi}=3$ in our experiment.

\section{CONCLUSION}

In this paper, we described a user study investigating vignetting as a method to reduce VR sickness when using amplified head 
rotations. Our results indicate that neither velocity nor accelerationbased vignetting were able to reduce the effects of VR sickness for all participants, and instead lead to a significant increase in sickness symptoms for 11 out of 15 participants. The majority of the participants preferred, and scored better in, the control condition without vignetting. This result stands in contrast to related work, where vignetting showed strong benefits when it was used with a different form of input using hand-held controllers, which we believe may be the main cause of the observed differences.

In future work, we plan to investigate the differences between velocity and acceleration-based masking methods, such as vignetting, when hand-held controllers are used separately or in concert with natural head movements. Moreover, we plan to look into differences in simulator sickness caused by up-scaled or down-scaled visual self-motion feedback and their relation to optic flow. Further, we plan to look into the characteristics of those users who benefit from vignetting to improve our understanding of individual differences in VR sickness.

\section{ACKNOWLEDGMENTS}

The work presented in this publication was supported in part by National Science Foundation (NSF) under Dr. Ephraim P. Glinert, Program Manager (NSF award 1564065) and the Office of Naval Research (ONR) code 30 under Dr. Peter Squire, Program Manager (ONR award N00014-17-1-2927), and in part by the Florida Hospital Endowed Chair in Healthcare Simulation.

\section{REFERENCES}

F. Biocca. 1992. Will simulation sickness slow down the diffusion of virtual environment technology? Presence: Teleoperators \& Virtual Environments 1, 3 (1992), 334-343.

M. Bolas, J A. Jones, I. McDowall, and E. Suma. 2017. Dynamic field of view throttling as a means of improving user experience in head mounted virtual environments. (May 9 2017). US Patent 9,645,395.

B. Bolte, G. Bruder, F. Steinicke, K. H. Hinrichs, and M. Lappe. 2010. Augmentation Techniques for Efficient Exploration in Head-Mounted Display Environments. In Proc. of the ACM Symposium on Virtual Reality Software and Technology. 11-18.

F. Bonato, A. Bubka, S. Palmisano, D. Phillip, and G. Moreno. 2008. Vection change exacerbates simulator sickness in virtual environments. Presence: Teleoperators and Virtual Environments 17, 3 (2008), 283-292.

P. Budhiraja, M. R. Miller, A. K. Modi, and D. A. Forsyth. 2017. Rotation Blurring Use of Artificial Blurring to Reduce Cybersickness in Virtual Reality First Person Shooters. CoRR abs/1710.02599 (2017).

Y. Farmani and R. J. Teather. 2018. Viewpoint Snapping to Reduce Cybersickness in Virtual Reality. In Proc. of Graphics Interface. 1-8.

A. S. Fernandes and S. K. Feiner. 2016. Combating VR sickness through subtle dynamic field-of-view modification. In Proc. of the IEEE Symposium on 3D User Interfaces. 201-210.

L. H. Frank, R. S. Kennedy, M. E. McCauley, R. W. Root, and R. S. Kellogg. 1984. Simulator sickness: Sensorimotor disturbances induced in flight simulators. Technical Report. Naval Training Equipment Center Orlando, FL.

C. Jay and R. Hubbold. 2003. Amplifying head movements with head-mounted displays Presence: Teleoperators \& Virtual Environments 12, 3 (2003), 268-276.

P. Kellnhofer, P. Didyk, K. Myszkowski, M.M. Hefeeda, H.-P. Seidel, and W. Matusik. 2016. GazeStereo3D: Seamless Disparity Manipulations. ACM Trans. Graph. 35, 4 (2016).

R.S. Kennedy, N.E. Lane, K.S. Berbaum, and M.G. Lilienthal. 1993. Simulator Sickness Questionnaire: An Enhanced Method for Quantifying Simulator Sickness. The International fournal of Aviation Psychology 3, 3 (1993), 203-220.

R. S. Kennedy, K. M. Stanney, and W. P. Dunlap. 2000. Duration and Exposure to Virtual Environments: Sickness Curves During and Across Sessions. Presence: Teleoperators and Virtual Environments 9, 5 (2000), 463-472.

R. Kopper, C. Stinson, and D. Bowman. 2011. Towards an understanding of the effects of amplified head rotations. In Proc. of the IEEE VR Workshop on Perceptual Illusions in Virtual Environments.

G. A. Koulieris, G. Drettakis, D. Cunningham, and K. Mania. 2016. Gaze Prediction using Machine Learning for Dynamic Stereo Manipulation in Games. In Proc. of IEEE Virtual Reality. 113-120.
J. J. LaViola, Jr., D. A. Feliz, D. F. Keefe, and R. C. Zeleznik. 2001. Hands-Free Multi-Scale Navigation in Virtual Environments. In Proc. of the Symposium on Interactive 3D Graphics. 26-29.

JJ-W Lin, H. B.-L. Duh, D. E. Parker, H. Abi-Rached, and T. A. Furness. 2002. Effects of field of view on presence, enjoyment, memory, and simulator sickness in a virtual environment. In Proc. of IEEE Virtual Reality. 164-171.

M. E. McCauley and T. J. Sharkey. 1992. Cybersickness: Perception of Self-motion in Virtual Environments. Presence: Teleoperators and Virtual Environments 1, 3 (1992), 311-318.

R. R. Mourant and T. R. Thattacherry. 2000. Simulator Sickness in a Virtual Environments Driving Simulator. Proc. of the Human Factors and Ergonomics Society Annual Meeting 44, 5 (2000), 534-537.

N. C. Nilsson, T. C. Peck, G. Bruder, E. Hodgson, S. Serafin, E. Suma, M. Whitton, and F. Steinicke. 2018. 15 Years of Research on Redirected Walking in Immersive Virtual Environments. IEEE Computer Graphics and Applications (2018), 1-19.

E. Patrick, D. Cosgrove, A. Slavkovic, J. A Rode, T. Verratti, and G. Chiselko. 2000. Using a large projection screen as an alternative to head-mounted displays for virtual environments. In Proc. of the ACM SIGCHI Conference on Human Factors in Computing Systems. 478-485.

T.C. Peck, M.C. Whitton, and H. Fuchs. 2008. Evaluation of Reorientation Techniques for Walking in Large Virtual Environments. In Proc. of IEEE Virtual Reality (VR). 121-128.

J. Plouzeau, D. Paillot, J Chardonnet, and F. Merienne. 2015. Effect of proprioceptive vibrations on simulator sickness during navigation task in virtual environment. In Proc. of the International Conference on Artificial Reality and Telexistence and the Eurographics Symposium on Virtual Environments. 1-6.

J. D. Prothero. 1995. Widening the field of view increases the sense of presence within immersive virtual environments. Technical Report. University of Washington.

E. D. Ragan, S. Scerbo, F. Bacim, and D. A. Bowman. 2017. Amplified Head Rotation in Virtual Reality and the Effects on 3D Search, Training Transfer, and Spatial Orientation. IEEE Transactions on Visualization and Computer Graphics 23, 8 (2017), $1880-1895$.

S. Razzaque, Z. Kohn, and M. Whitton. 2001. Redirected Walking. In Proc. of Eurographics. 289-294.

S. Razzaque, D. Swapp, M. Slater, M. C. Whitton, and A. Steed. 2002. Redirected Walking in Place. In Proc. of the Eurographics Workshop on Virtual Environments. 123-130.

J. T. Reason and J. J. Brand. 1975. Motion Sickness. Academic Press.

L. Rebenitsch and C. Owen. 2014. Individual variation in susceptibility to cybersickness. In Proc. of the ACM Symposium on User Interface Software and Technology. 309-317.

E. Ressiot, M. Dolz, L. Bonne, and R. Marianowski. 2013. Prospective study on the efficacy of optokinetic training in the treatment of seasickness. European Annals of Otorhinolaryngology, Head and Neck Diseases 130, 5 (2013), 263-268.

G. E Riccio and T. A Stoffregen. 1991. An ecological theory of motion sickness and postural instability. Ecological psychology 3, 3 (1991), 195-240.

W. Robinett and R. Holloway. 1992. Implementation of Flying, Scaling and Grabbing in Virtual Worlds. In Proc. of the ACM Symposium on Interactive 3D Graphics. 189-192.

S. P. Sargunam, K. Rahimi Moghadam, M. Suhail, and E. D. Ragan. 2017. Guided head rotation and amplified head rotation: Evaluating semi-natural travel and viewing techniques in virtual reality. In Proc. of IEEE Virtual Reality (VR). 19-28.

M. J. Schuemie, P. van der Straaten, M. Krijn, and C. A. P. G. van der Mast. 2001. Research on presence in virtual reality: a survey. Cyberpsychology and Behaviour 4, 2 (2001), 183-202.

S. Sharples, S. Cobb, A. Moody, and J. R Wilson. 2008. Virtual reality induced symptoms and effects (VRISE): Comparison of head mounted display (HMD), desktop and projection display systems. Displays 29, 2 (2008), 58-69.

M. Slater, M. Usoh, and A. Steed. 1994. Depth of Presence in Virtual Environments. Presence: Teleoperators and Virtual Environments 3, 2 (1994), 130-144.

M. Slater, M. Usoh, and A. Steed. 1995. Taking steps: the influence of a walking technique on presence in virtual reality. ACM Transactions on Computer-Human Interaction 2, 3 (1995), 201-219.

K.M. Stanney and R.S. Kennedy. 1997. The Psychometrics of Cybersickness. Commun. ACM 40, 8 (1997), 66-68.

K. M. Stanney, R. S. Kennedy, and J. M. Drexler. 1997. Cybersickness is not simulator sickness. In Proc. of the Human Factors and Ergonomics Society Annual Meeting, Vol. 41. 1138-1142.

F. Steinicke, G. Bruder, J. Jerald, H. Fenz, and M. Lappe. 2010. Estimation of Detection Thresholds for Redirected Walking Techniques. IEEE Transactions on Visualization and Computer Graphics (TVCG) 16, 1 (2010), 17-27.

F. Steinicke, Y. Visell, J. Campos, and A. Lecuyer. 2013. Human Walking in Virtual Environments: Perception, Technology, and Applications. Springer Verlag.

N. Teasdale, M. Lavallière, M. Tremblay, D. Laurendeau, and M. Simoneau. 2009. Multiple exposition to a driving simulator reduces simulator symptoms for elderly drivers. In Proc. of the International Driving Symposium on Human Factors in Driver Assessment, Training and Vehicle Design.

M. Usoh, E. Catena, S. Arman, and M. Slater. 2000. Using Presence Questionnaires in Reality. Presence: Teleoperators and Virtual Environments 9, 5 (2000), 497-503.

R. Zhang and S. A. Kuhl. 2013. Human Sensitivity to Dynamic Rotation Gains in Head-mounted Displays. In Proc. of the ACM Symposium on Applied Perception. $71-74$. 